\title{
Pulmonary eosinophilia associated with montelukast
}

\author{
José Franco, María José Artés
}

\begin{abstract}
Antileukotriene drugs are new therapeutic agents that have recently been approved for the treatment of asthma. Several cases of eosinophilic conditions including ChurgStrauss syndrome have been reported to be associated with zafirlukast, a cysteinyl leukotriene type 1 receptor antagonist. So far no other leukotriene modifier has been associated with the syndrome. The case history is presented of a man with allergic rhinitis and asthma who had received intermittent pulse therapy with oral corticosteroids. Pulmonary eosinophilia developed while he was receiving treatment with montelukast, a chemically distinct cysteinyl leukotriene type 1 receptor antagonist. After discontinuation of montelukast therapy and administration of systemic corticosteroids the patient's symptoms reversed rapidly and there was prompt resolution of the pulmonary infiltrates. We believe that cysteinyl leukotriene type 1 receptor antagonists are safe and effective drugs for most patients with asthma but caution is needed for those with more severe disease who require systemic corticosteroids, especially if they show characteristics of the atypical allergic diathesis seen in the prodromal phase of Churg-Strauss syndrome. (Thorax 1999;54:558-560)
\end{abstract}

Keywords: montelukast; side effects; pulmonary eosinophilia

Department of

Pneumology

J Franco

Department of Pathology

M J Artés

University Hospital Dr. Peset, Valencia, Spain

Correspondence to: Dr J Franco, Diputado Luis Lucía 19-5, E-46015 Valencia, Spain.

Received 6 January 1999 Returned to authors 26 February 1999 Revised manuscript received 12 March 1999 Accepted for publication 16 March 1999

The antileukotriene drugs are new therapeutic agents which have recently been approved and are now available for the treatment of asthma in several countries. These drugs include one enzyme inhibitor of 5-lipoxygenase, zileuton, and three chemically distinct cysteinyl leukotriene type 1 receptor antagonists, zafirlukast, pranlukast and montelukast. ${ }^{1}$

Although clinical studies have shown that antileukotriene drugs are safe and effective, the results do not yet provide guidelines for their optimal clinical use in the treatment of asthma. ${ }^{1}$ As the use of these drugs increases, adverse events occurring at low frequency or in populations not examined in clinical trials may become manifest. ${ }^{2}$ Several cases of eosinophilic conditions including Churg-Strauss syndrome have been reported in patients who have been treated with zafirlukast. ${ }^{2-4}$ No other leukotriene modifier has yet been associated with the syndrome.

In this case report we describe an asthmatic patient in whom pulmonary eosinophilia developed while receiving montelukast therapy.

\section{Case report}

A 26 year old man with a three year history of asthma had received treatment with salbutamol, nedocromil, theophylline, and beclomethasone. There was a five year history of allergic rhinitis with positive skin tests for house dust mite and cat dander. Because asthma symptoms were not well controlled, treatment was started with fluticasone $1000 \mu \mathrm{g}$ daily and salmeterol twice a day. Allergen immunotherapy was also prescribed. Nonetheless, short courses of oral prednisone $40 \mathrm{mg}$ or deflazacort $60 \mathrm{mg}$ daily were required on several occasions to control asthma exacerbations. A decision was made to initiate treatment with montelukast at a dose of $10 \mathrm{mg}$ daily in the evening. Treatment with fluticasone was continued.

After approximately four months of treatment with montelukast the patient developed headache, malaise, myalgia, nasal congestion, and fever up to $39^{\circ} \mathrm{C}$. Oral cefuroxime and paracetamol were prescribed. Seven days later the patient developed dyspnoea and was admitted to our hospital.

On physical examination there were diffuse wheezes and ronchi. No cutaneous lesions or arthritis were found. A chest radiograph (fig 1) showed mixed alveolar-interstitial infiltrates in the upper lobes. Laboratory findings demon-

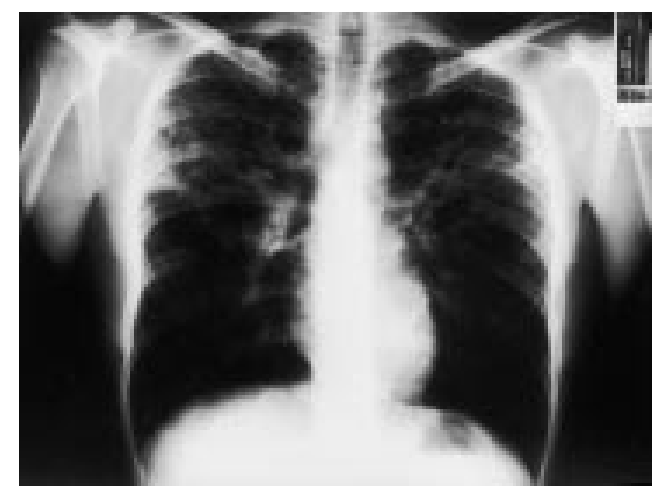

Figure 1 Posteroanterior radiograph of the chest showing mixed alveolar-interstitial infiltrates in both upper lobes. 


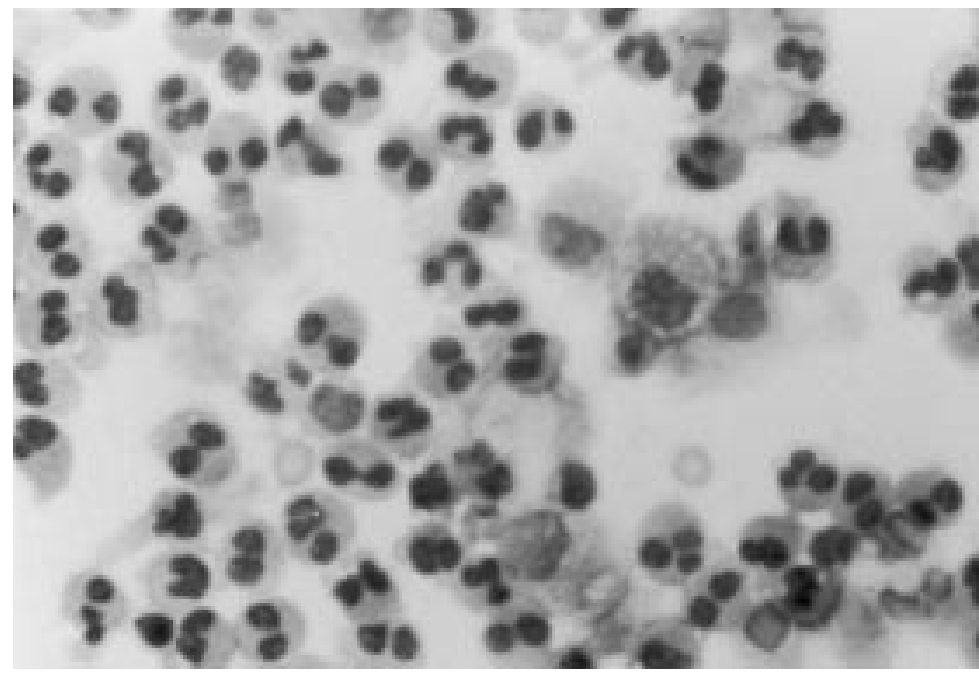

Figure 2 Bronchoalveolar lavage fluid showing a large number of eosinophils as well as scant macrophages. Papanicolaou stain; $\times 40$.

with an eosinophil fraction of 0.14 ; the total IgE level was $527 \mathrm{IU} / 1$ and the results of an antineutrophil cytoplasmic antibody (ANCA) test were negative. A specimen of arterial blood, drawn while the patient was breathing room air, had a $\mathrm{pH}$ of 7.45 with oxygen tension $\left(\mathrm{PaO}_{2}\right)$ of $8.7 \mathrm{kPa}$ and carbon dioxide tension $\left(\mathrm{PaCO}_{2}\right)$ of $5.0 \mathrm{kPa}$. A fibreoptic bronchoscopic examination found no endobronchial lesions. Bronchoalveolar lavage fluid (fig 2) contained macrophages $(10 \%)$, lymphocytes $(10 \%)$, and eosinophils $(80 \%)$. No acid-fast bacilli, fungi, or parasites were seen in the lavage fluid. Microscopic examination of a stool specimen disclosed no parasites.

Montelukast treatment was discontinued and treatment with intravenous methylprednisolone at a dosage of $60 \mathrm{mg}$ daily was begun. Both dyspnoea and constitutional symptoms improved within a short time. After nine days of treatment with high dose systemic corticosteroids a chest radiograph revealed resolution of pulmonary infiltrates.

\section{Discussion}

Montelukast is a potent and selective cysteinyl leukotriene type 1 receptor antagonist with potency and oral activity similar to those of zafirlukast. ${ }^{5}$ At a dosage of $10 \mathrm{mg}$ montelukast inhibits bronchospasm after allergen or exercise challenge and causes clinically significant improvement in patients with asthma. The tolerability profile of montelukast was similar to that of placebo in placebo controlled clinical trials in adults and children, and the most common adverse effect reported was headache. ${ }^{6}$

In the case reported here the temporal relationship between montelukast treatment and the development of pulmonary eosinophilia suggests a causal association. However, we cannot exclude the possibility that the patient might have developed another eosinophilic condition such as allergic bronchopulmonary aspergillosis even without treatment with montelukast. The absence of parasites in the stools and the bronchoscopic specimens makes parasite induced eosinophilic lung disease unlikely.
Despite the lack of systemic vasculitis and a negative ANCA, this patient with his five year history of allergic rhinitis, progressive airways symptoms, and intermittent treatment with oral corticosteroids could have a forme frustre of Churg-Strauss syndrome.

Wechsler et al reported eight cases of Churg-Strauss syndrome following discontinuation of treatment with oral corticosteroids in patients with asthma receiving zafirlukast. The authors speculated that all these patients suffered from a primary eosinophilic infiltrative disorder that was unmasked when steroids were withdrawn. However, in two other cases of Churg-Strauss syndrome associated with zafirlukast therapy ${ }^{3}$ and in the case of pulmonary eosinophilia reported in this paper there was no association between corticosteroid withdrawal and the development of the syndrome. Because all these patients had moderate to severe asthma and had received intermittent or regular treatment with oral corticosteroids, they could have been formes frustres of Churg-Strauss syndrome whose manifestations were quelled by prior steroid treatment. ${ }^{7}$

As this syndrome has not yet been seen in patients treated with zileuton, a drug that inhibits leukotriene B4 (LTB4) and cysteinyl leukotrienes (LTC4, LTD4 and LTE4), an alternative explanation based on the imbalance in leukotriene receptor stimulation has been put forward. ${ }^{8}$ Cysteinyl leukotriene type 1 receptor antagonism may have led to increased circulating LTB4 which has been shown to be a chemoattractant for eosinophils as well as neutrophils. ${ }^{9}$

Pulmonary eosinophilia associated with a cysteinyl leukotriene type 1 receptor antagonist other than zafirlukast suggests that the syndrome may be related to the effect of antileukotriene drugs on leukotriene receptors.

Churg-Strauss syndrome is a disorder characterised by hypereosinophilia and systemic vasculitis which occurs in a subset of patients with asthma and allergic rhinitis whose allergic diathesis is atypical with respect to the late age of onset of symptoms, frequent absence of family history, severity of upper respiratory tract disease, and exaggerated eosinophil response. ${ }^{10}$ The patient described here and the cases of Churg-Strauss syndrome associated with zafirlukast previously reported could belong to this category of patients. We hypothesise that, in such individuals, cysteinyl leukotriene type 1 receptor antagonists can precipitate the phase of the illness characterised by eosinophilic tissue infiltrates or life threatening systemic vasculitis.

We believe that cysteinyl leukotriene type 1 receptor antagonists remain safe and effective drugs for most patients with mild to moderate asthma but, while the exact causes of the syndrome are not well understood, caution is needed for those with more severe disease who require systemic corticosteroids, especially if they show characteristics of the atypical allergic diathesis seen in the prodromal phase of Churg-Strauss syndrome. 
1 O'Byrne PO, Israel E, Drazen JM. Antileukotrienes in the treatment of asthma. Ann Intern Med 1997;127:47280.

2 Wechsler ME, Garpestad E, Flier SR, et al. Pulmonary infiltrates, eosinophilia, and cardiomyopathy following corticosteroid withdrawal in patients with asthma receiving zafirlukast. $¥ A M A$ 1998;279:455-7

3 Katz RS, Papernik M. Zafirlukast and Churg-Strauss syndrome. ҒAMA 1998;279:1949.

4 Knoell DL, Lucas J, Allen JN. Churg-Strauss syndrome associated with zafirlukast. Chest 1998;114:332-4.

5 Aharony D. Pharmacology of leukotriene receptor antago- nists. Am $\mathcal{F}$ Respir Crit Care Med 1998;157:s214-9.

6 Markham A, Faulds D. Montelukast. Drugs 1998;56:251-6.

7 Churg J, Churg A. Zafirlukast and Churg-Strauss syndrome. $\mathcal{F A M A}$ 1998;279:1949-50.

8 Honsinger RW. Zafirlukast and Churg-Strauss syndrome. FAMA 1998;279:1949.

9 Crooks SW, Stockley RA. Leukotriene B4. Int $\mathcal{F}$ Biochem Cell Biol 1998;30:173-8.

10 Lanham JG, Elkon KB, Pusey CD, et al. Systemic vasculitis with asthma and eosinophilia: a clinical approach to the Churg-Strauss syndrome. Medicine 1984;63:65-81.

\title{
Thorax 1999;54:560-561 Epithelioid haemangioendothelioma
}

\author{
M J Ledson, R Convery, A Carty, C C Evans
}

\begin{abstract}
Epithelioid haemangioendothelioma is a rare pulmonary neoplasm with less than 40 cases described world wide. We describe the only case to have presented with hypertrophic pulmonary osteoarthropathy who has been treated with azathioprine and has remained alive and well with no deterioration in pulmonary function since being diagnosed 16 years ago. The progression of the chest radiograph and spiral CT appearances of this rare neoplasm are described, and current views regarding the cellular origin of the neoplasm, its cytological appearance, clinical presentation and prognosis are discussed.
\end{abstract}

(Thorax 1999;54:560-561)

Keywords: epithelioid haemangioendothelioma; intravascular sclerosing bronchioalveolar tumour

Epithelioid haemangioendothelioma (EHE) (formerly known as intravascular sclerosing bronchioalveolar tumour, IVBAT) is a rare pulmonary neoplasm with less than 40 cases described world wide. We have described the first reported case presenting with hypertrophic pulmonary osteoarthropathy. ${ }^{1}$ We now report the progress of this patient who is unique in that she has been treated with azathioprine and has remained alive and well with no deterioration in pulmonary function since being diagnosed 16 years ago.

\section{Case report}

The Cardiothoracic Centre, Thomas Drive, Liverpool L14 3PE, UK M J Ledson

R Convery

A Carty

C C Evans

Correspondence to: Dr C C Evans.

Received 14 July 1997 Returned to author 12 September 1997 Revised manuscript received 5 November 1997 Accepted for publication 20 November 1997
In April 1981 a 24 year old woman presented to our unit with a six month history of pain and swelling in her ankles and a six week history of mild exertional dyspnoea. On examination her fingers and toes were grossly clubbed and her wrists and ankles were swollen and tender. Inspiratory crackles were audible at the left base posteriorly. Her chest radiograph, which had been normal six years earlier, showed multiple irregular, unequal, bilateral, uniformly distributed, non-calcified nodular shadowing. Radiographs of her wrists and ankles showed changes of hypertrophic pulmonary osteoarthropathy. Full blood count, urea and electrolytes, liver function tests, calcium, and serum

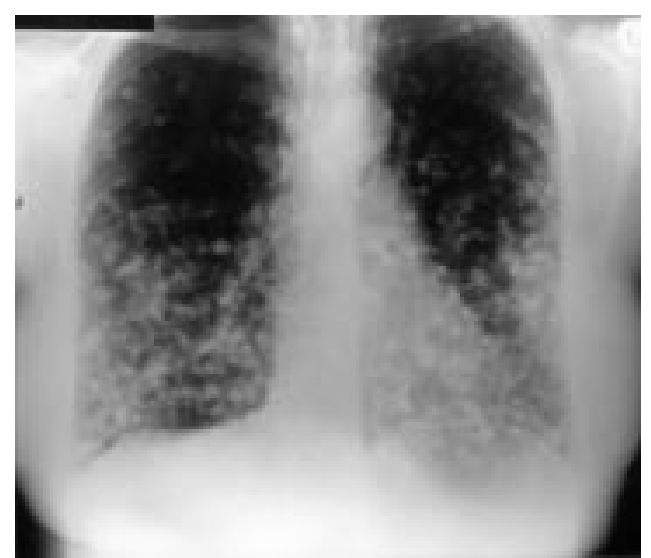

Figure 1 Chest radiograph in 1996 revealing multiple irregular unequal bilateral calcified uniformly distributed nodular shadowing.

immunoglobulins were all normal. Autoantibodies including rheumatoid factor and antinuclear factor were negative, as was a Mantoux test. The erythrocyte sedimentation rate was $46 \mathrm{~mm}$ in the first hour. Pulmonary function tests revealed a mild restrictive ventilatory defect with lung volumes $70 \%$ predicted. Gas transfer was normal. No endobronchial lesions were found at fibreoptic bronchoscopy and a transbronchial lung biopsy specimen was unhelpful. The patient therefore underwent an open lung biopsy via a left thoracotomy. At operation multiple nodules up to $15 \mathrm{~mm}$ in diameter were found throughout the lung. Two wedge biopsies were taken from the lingula. All nodules had similar histological appearances which were considered typical of EHE.

As the majority of her symptoms were due the hypertrophic pulmonary osteoarthropathy indomethacin was commenced with good effect. Azathioprine was also started and the patient returned to work. The patient has been reviewed yearly for the past 16 years and her symptoms have remained unchanged. Increasing calcification of the nodules was seen radiologically by 1989, and by 1996 (fig 1) the overall number of nodules remained unchanged but their size had decreased to a maximum of $12 \mathrm{~mm}$. A thoracic spiral intravenously enhanced CT scan was performed in 1997 (fig 2) showing innumerable $12 \mathrm{~mm}$ calcified nodules in all areas but more concentrated at the lung 


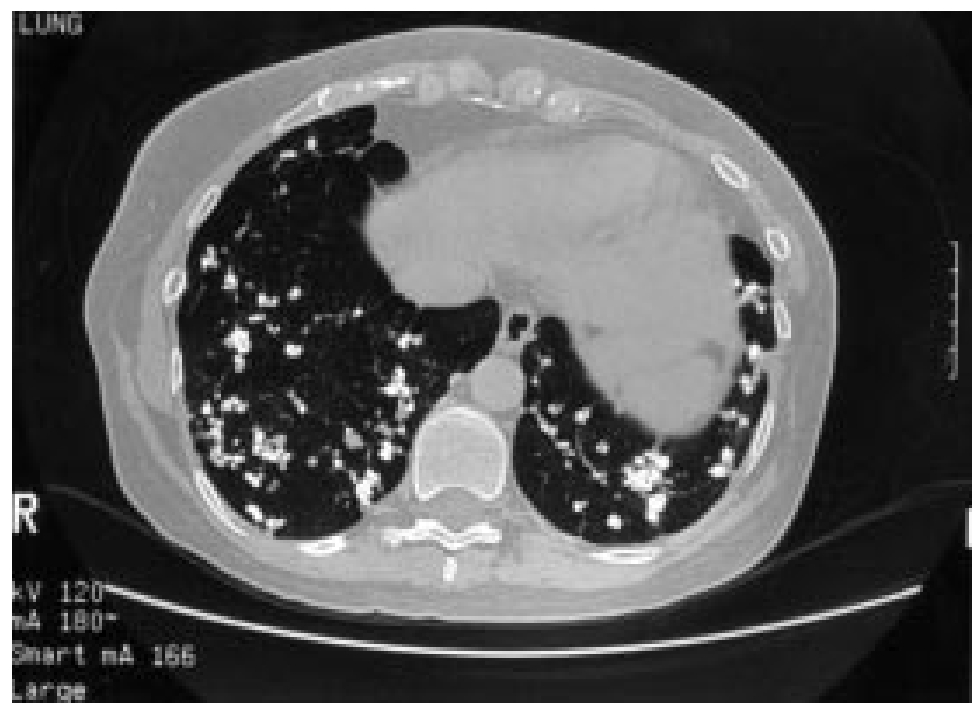

Figure 2 Spiral CT scan (enhanced with contrast) showing innumerable calcified nodules and diffuse fibrosis in both lungs.

bases and subpleurally. This CT scan also revealed about 20 low attenuation, nonenhancing, asymmetrically calcified liver lesions. In October 1995 azathioprine was discontinued. Her pulmonary function tests have not deteriorated over the past 16 years.

\section{Discussion}

EHE is a rare pulmonary neoplasm with fewer than 40 cases reported in the literature. The tumour was initially described in 1975 by Dail and Liebow and was named intravascular sclerosing bronchioalveolar tumour (IVBAT) ${ }^{23}$ because of the proposed bronchioalveolar origin of the neoplasm. Corrin $e t a l^{4}$ postulated that the tumour arose from precursor cells capable of differentiation along endothelial cell lines. Weldon-Linne et al, ${ }^{5}$ using electron microscopy, determined endothelial characteristics of the cells and thought they were derived from multipotential mesenchymal reserve cells. Using applied immunochemistry, Bhagavan et $a l^{6}$ described diffuse cytoplasmic staining of tumour cells with factor VIII related antigen which binds to a storage product of endothelial cells.

Microscopically the initial change is an interstitial alveolar infiltrate with lymphocytes, followed by protrusion of the alveolar wall in a polypoidal manner into the alveolar lumen. This protrusion is covered with hypertrophied alveolar epithelial cells and its core consists at first of a mixoid type of connective tissue which stains positively with PAS and Congo Red. As the stroma matures it is converted into hyaline fibrous tissue. Embedded in this hyaline fibrous tissue are large malignant-appearing cells with large round to oval grainy nuclei, often prominent nucleoli, and abundant homogeneous cytoplasm. Eventually the whole alveolus is obliterated. The same tissue that obliterates the alveoli may spread into and along the small bronchi and bronchioles and also fills small branches of the pulmonary arteries and veins. ${ }^{7}$ Thus multiple non-encapsulated parenchymal nodules are seen exhibiting a zonal architecture. There have been reports of nodules with calcifi- cation, chondrification, or even ossification, but this occurs in less than $10 \%$ of cases. ${ }^{3}$

Weiss et $a l^{8}$ first used the term EHE. It is now well recognised that the IVBAT is an EHE occurring in the lung which may also occur in the soft tissues, bone, and liver.

Three quarters of patients with EHE of the lung are women. The age range at presentation is wide (12-61 years), and $40 \%$ of patients are below the age of 30 . Half the patients are asymptomatic and are discovered on incidental chest radiography. Symptoms are uncommon and are usually mild and include shortness of breath, mild pleuritic chest pain, and nonproductive cough, although haemoptysis has been reported. However, hypertrophic pulmonary osteoarthropathy has not been previously reported. The chest radiograph usually reveals multiple bilateral pulmonary nodules and pleural effusions are rarely seen. ${ }^{9}$ Distant metastases are seen in less than a quarter of cases, the sites involved including the liver, lymph nodes, bowel, retroperitoneal soft tissues, and skin. In most cases the disease has a slowly progressive course and patients die 2-24 years after diagnosis of restrictive lung disease. The shortest survival is eight weeks in a patient who succumbed to gross haemoptysis. ${ }^{10}$ The longest survivor was treated surgically, undergoing 11 separate resections over 24 years, finally succumbing to pneumonia superimposed on a decreasing respiratory function. ${ }^{11}$ Other treatments tried have been chemotherapy and radiotherapy with limited success in symptomatic patients near death. Our patient refused the empirical use of oral corticosteroids in an attempt to control potential fibrosis. She was therefore commenced on azathioprine following diagnosis and so far has had no deterioration in pulmonary function. However, at the patient's request azathioprine was discontinued in October 1995. The patient is now the second longest survivor with this condition. She continues to have mild symptoms from her hypertrophic pulmonary osteoarthropathy but is asymptomatic from the pulmonary point of view and is still able to work as a paediatric nurse.

1 Marsh K, Kenyon WE, Earis JE, et al. Intravascular bronchioalveolar tumour. Thorax 1982;37:474-5.

2 Dail D, Liebow A. Intravascular bronchioalveolar tumour (abstract). F Pathol 1975;78:6-7.

3 Dail DH, Liebow AA, Gmelich JT, et al. Intravascular bronchiolar and alveolar tumour of the lung (IVBAT): an analysis of twenty cases of a peculiar sclerosing endothelial tumour. Cancer 1983;51:452-64.

4 Corrin B, Manners B, Millard M, et al. Histiogenesis of the so called "intravascular bronchioalveolar tumour". F Pathol 1979; 128:163-7.

5 Weldon-Linne CM, Victor TA, Christ ML, et al. Angiogenic nature of the "intravascular bronchioalveolar tumour" of the lung: an electron microscopic study. Arch Pathol Lab Med 1981;105:174-9.

6 Bhagavan BS, Eggleston JC, Dorfman HD, et al. Intravascular bronchioalveolar tumour (IVBAT). Cancer 1983;52: 452-64.

7 Spencer H. Pathology of the lung. 3rd edn. Oxford: Pergamon Press, 1977: 907-9.

8 Weiss SW, Ishak KG, Dail DH, et al. Epitheliod haemangioendothelioma and related lesions. Semin Diagn Pathol 1986;3:259-87.

9 Buggage RR, Soudi N, Olson JL, et al. Epithelioid haemangioendothelioma of the lung. Diagn Cytopathol 1995;13:

10 Carter EJ, Bradburne RM, Jhung JW, et al. Alveolar haemorrhage with epithelioid haemangioendothelioma. Am Rev Respir Dis 1990;142:700-1.

11 Miettinen M, Collan Y, Halttunen P, et al. Intravascular bronchoalveolar tumor. Cancer 1987;60:2471-5. 